\title{
Combined effects of acute exercise and hypoxia on memory
}

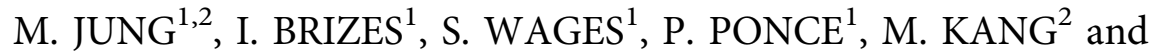 \\ P.D. LOPRINZI ${ }^{1 *}$
}

\begin{abstract}
${ }^{1}$ Exercise \& Memory Laboratory, Department of Health, Exercise Science and Recreation Management, The University of Mississippi, University, MS 38677, USA

${ }^{2}$ Health and Sport Analytics Laboratory, Department of Health, Exercise Science and Recreation Management, The University of Mississippi, University, MS 38677, USA
\end{abstract}

Received: March 28, 2020 • Accepted: May 27, 2020

Published online: July 02, 2020

(C) 2020 Akadémiai Kiadó, Budapest

\begin{abstract}
No previous studies have evaluated the potential combined effects of acute exercise and acute hypoxia exposure on memory function, which was the purpose of this study. Twenty-five participants $\left(\mathrm{M}_{\text {age }}=21.2\right.$ years) completed two laboratory visits in a counterbalanced order, involving 1) acute exercise (a 20-min bout of moderate-intensity exercise) and then $30 \mathrm{~min}$ of exposure to hypoxia $\left(\mathrm{FIO}_{2}=0.12\right)$, and 2 ) exposure to hypoxia alone $\left(\mathrm{FIO}_{2}=0.12\right)$ for $30 \mathrm{~min}$. Following this, participants completed a cued-recall and memory interference task ( $\mathrm{AB} / \mathrm{AC}$ paradigm), assessing cued-recall memory (recall 1 and recall 2 ) and memory interference (proactive and retroactive interference). For cued-recall memory, we observed a significant main effect for condition, with Exercise + Hypoxia condition having significantly greater cuedrecall performance than Hypoxia alone. Memory interference did not differ as a function of the experimental condition. This experiment demonstrates that engaging in an acute bout of exercise prior to acute hypoxia exposure had an additive effect in enhancing cued-recall memory performance.
\end{abstract}

\section{KEYWORDS}

cognition, cognitive function, synaptic plasticity

\footnotetext{
* Corresponding author. Exercise \& Memory Laboratory, Department of Health, Exercise Science and Recreation Management, The University of Mississippi, University, MS 38677, USA. Tel.: +1 662915 5561,

E-mail: pdloprin@olemiss.edu
} 


\section{INTRODUCTION}

Populations that are repeatedly exposed to a hypoxic environment (e.g., mountaineers rescuers and the armed forces) may be at high risk of cognitive dysfunction. Ascending to higher altitudes and the ensuing hypoxia may interfere with the central nervous system (CNS), neurological and/or physiological function, due to an insufficient supply of oxygen to the brain tissue $[1,2]$. Such effects may compromise cognitive abilities, including executive function, episodic memory, and memory interference. Executive function is considered a higher-order cognition involving aspects of working memory, cognitive inhibition, and cognitive flexibility. Episodic memory refers to the retrospective recall of information, typically in a spatiotemporal context. Lastly, interference-based forgetting paradigms involve competing information occurring before (proactive interference, PI), during (concurrent; dual-task paradigms), or after (retroactive interference, RI) the target learning event. For example, knowing an acquaintance's maiden name may proactively interfere with the retention of their married surname. On the other hand, long-term knowledge of a friend's married surname may retroactively interfere with the recall of the friend's maiden name.

A number of studies have investigated the relationship between hypoxia and cognitive function and demonstrated a negative effect [3-5]. For example, a systematic review by McMorris et al. [5] indicated that acute hypoxia ( $<60 \mathrm{mmHg}$ for $10 \mathrm{~min}$ to 5 days) was associated with worse cognition (both central executive and non-executive tasks). An experimental study by de AquinoLemos et al. [3] showed that exposure of an acute $(90 \mathrm{~min}$ ) hypoxic condition (simulated altitude of $4500 \mathrm{~m}$ ) impaired reaction time. This, however, is in contrast to a recent publication [4], which demonstrated that acute hypoxia had a favorable effect on memory. Specifically, exposure to an acute bout (30 $\mathrm{min}$ ) of hypoxia at an altitude equivalent of $4000 \mathrm{~m}$ was shown to be sufficient in attenuating memory interference. Given the conflicting findings in prior studies, such therapeutic or detrimental effects of acute hypoxia on cognition (e.g., memory) appear to be reliant on the dose of hypoxia (e.g., severity and duration of hypoxic episodes).

As thoroughly discussed elsewhere [6], high dose of acute hypoxia triggers unfavorable pathologies, including hippocampal apoptosis, impaired synaptic plasticity, and cognitive impairment [7]. On the other hand, low dose of acute hypoxia elicits improved respiratory and non-respiratory somatic motor function [8] and increased growth factor expression in the CNS [9]. Moreover, while prolonged exposure to hypoxia leads to decreased regional cerebral metabolism and reduced activation in brain areas (e.g., angular gyrus, prefrontal cortex, and thalamus) associated with cognition [10], acute intermittent hypoxia (i.e., 15-75 min of hypoxia exposure) may facilitate neuroprotection and neuroplasticity [6]. Although speculative, the mechanisms of these beneficial effects (low-dose and single exposure of acute hypoxia) may be related to brain-derived neurotrophic factor (BDNF) and vascular endothelial growth factor (VEGF). Increased production of BDNF and VEGF play a key role in subserving memory function $[11,12]$. Tropomyosin-regulated kinase B (TrkB), a BDNF main receptor, is regulated by hypoxia-inducible factor-1, a transcription factor regulated by hypoxia-sensitive genes [13]. Therefore, TrkB is upregulated in the whole brain following acute hypoxia. These acute hypoxia effects may promote BDNF-dependent synaptic plasticity and hippocampal neurogenesis [14]. Similarly, VEGF contributes to the regulation of hippocampal neurogenesis and long-term memory formation via increased hypoxia-inducible factor-1 [15]. As such, hypoxia-induced production of these two key growth factors appears to play a critical role in enhancing memory function. On the other hand, however, and as stated, high dose of acute hypoxia triggers 
unfavorable effects, including hippocampal apoptosis, impaired synaptic plasticity, and ultimately, cognitive impairment. Further, high doses or prolonged exposure to hypoxia may elevate levels of BDNF to an excessively high level, which may promote hyperexcitability and/or excitotoxic damage [16].

In addition to hypoxia intervention, acute exercise (single bout of exercise) has been shown to increase neural activity in many brain structures (e.g., prefrontal cortex and hippocampus) that improve memory performance; the exercise-related mechanisms of memory function have been discussed elsewhere $[17,18]$. Briefly, acute exercise may increase neural excitability and long-term potentiation in key memory-related brain structures $[19,20]$. Accumulating research demonstrates that acute exercise has beneficial effects on various memory functions (e.g., shortterm memory, working memory, and episodic long-term memory) [21, 22]. More recently, emerging work has been interested in experimentally evaluating the effects of acute exercise on memory interference $[23,24]$. For example, these prior studies have demonstrated that acute exercise can attenuate proactive interference (i.e., reducing a memory impairment effect from previously learned information) and retroactive interference (i.e., currently learned information impairing the recall of previously learned information). The mechanisms of this effect have been thoroughly discussed elsewhere [25]. In brief, previous studies have provided suggestive evidence that the medial prefrontal cortex (mPFC) and hippocampus, both of which are activated via acute exercise [26], play an integral role in reducing a memory interference effect [27]. For instance, $\mathrm{mPFC}$ not only minimizes the encoding of the interfering stimuli but also facilitates the consolidation of the initial stimuli [28]. The hippocampus facilitates retrieval of memory and pattern separation during the learning process [29]. Thus, although only a few published experiments have evaluated this possibility, acute exercise may help to attenuate a memory interference effect (both proactive and retroactive memory).

Couched within the above, acute hypoxia induces upregulation of growth/trophic factors in regions of the brain involved in memory function and acute exercise upregulates neural activity, including increased excitatory post-synaptic potentials in the hippocampus and BDNF expression, which play a significant role in subserving memory performance. Further, both acute hypoxia and exercise have been shown to attenuate memory interference [4, 23, 24]. As such, it is plausible that acute exercise combined with acute hypoxia may have a synergetic effect in improving memory and attenuating memory interference. This experiment is a follow-up to a recent publication [4], which demonstrated that acute hypoxia attenuated memory interference. To extend this previous work, we aimed to see if acute exercise coupled with acute hypoxia would result in greater memory performance and attenuate memory interference to a greater extent than acute hypoxia alone.

\section{METHODS}

All procedures were approved by the ethics committee at the University of Mississippi. All participants provided written consent prior to participation. See Table 1 (below) for the schematic of the study protocol. A within-subject design was implemented. The two conditions were counterbalanced, including exercise and then hypoxia, as well as hypoxia alone. We specifically implemented a within-subject posttest design, with the memory assessment occurring after the experimental manipulation for each condition. This approach, as opposed to a within-subject 
Table 1. Study protocol

\begin{tabular}{|c|c|c|c|c|c|}
\hline \multicolumn{3}{|c|}{$\begin{array}{l}\text { Exercise and then hypoxia } \\
\left(\mathrm{PIO}_{2}=87 \mathrm{mmHG}, \mathrm{FIO}_{2}=0.12,\right. \\
\text { simulated altitude of } 4000 \mathrm{~m})^{\mathrm{a}}\end{array}$} & \multicolumn{3}{|c|}{$\begin{array}{c}\text { Hypoxia alone } \\
\left(\mathrm{PIO}_{2}=87 \mathrm{mmHG}, \mathrm{FIO}_{2}=0.12\right. \\
\text { simulated altitude of } 4000 \mathrm{~m})^{\mathrm{a}}\end{array}$} \\
\hline $\begin{array}{c}20 \text {-min exercise } \\
\text { (50\% HRR) }\end{array}$ & $\begin{array}{l}\text { 30-min hypoxia } \\
\text { exposure }^{\mathrm{a}}\end{array}$ & $\begin{array}{l}\text { Memory } \\
\text { task }\end{array}$ & $\begin{array}{c}\text { Rest } \\
(20-\mathrm{min})\end{array}$ & $\begin{array}{c}\text { 30-min hypoxia } \\
\text { exposure }^{\mathrm{a}}\end{array}$ & $\begin{array}{c}\text { Memory } \\
\text { task }\end{array}$ \\
\hline
\end{tabular}

HRR, heart rate reserve.

${ }^{a}$ The 30-min exposure of acute hypoxia involved a gradual progression from $\mathrm{FIO}_{2}=0.21$ (21\%) to $\mathrm{FIO}_{2}=0.12(12 \%)$ over the first 5-min. That is, within the first $5 \mathrm{~min}$ of acute hypoxia exposure, there was a gradual progression to an $\mathrm{FIO}_{2}=0.12$, and then from minutes 5 to 30 , the $\mathrm{FIO}_{2}$ stayed at 0.12 .

pretest posttest design (memory assessment before and after the experimental manipulation for each condition), was implemented to avoid a potential proactive memory interference effect (i.e., the pretest memory task influencing the posttest memory results).

\section{Participants}

Similar to previous work [4, 30, 31], 25 participants completed both visits. A priori power calculations were conducted using $G^{\star}$ Power with the following parameters, looking at the within-subject effects for $2 \times 2$ repeated measures ANOVA: medium effect size $f=0.25$, alpha level $=0.05$, power $=0.8$, and correlation among repeated measures $=0.7$. This resulted in a recommended sample size of 22 for the within-subjects effect. Assuming $10 \%$ attrition, we recruited a total of 25 participants in this study.

All participants were naive to the protocol, with no previous exposure to the normobaric procedure or exposure to laboratory-induced hypoxia. All participants lived at an approximate altitude of $154 \mathrm{~m}$. Participants were excluded if they: 1) were outside the age range of 18-35 y, 2) exercised within $5 \mathrm{~h}$ of their visit, 3) consumed caffeine within $3 \mathrm{~h}$ of their visit, 4) were a daily smoker, 5) self-reported being pregnant, 6) took marijuana in the past 30 days, 7) had a diagnosis of $\mathrm{ADD} / \mathrm{ADHD}$ or taking medications for these conditions, 8) consumed more than 1 alcoholic drink/day (female) or more than 2 alcoholic drinks/day (male), 9) had a concussion in the past 30 days, 10) had a resting blood pressure $\geq 140 / 90 \mathrm{mmHg}, 11$ ) self-report a previous episode of altitude sickness, 12) self-reported or diagnosed asthma, or 13) had one or more of the following clotting risk factors: previously had a deep vein thrombosis (DVT), family history of DVT, previously had a thromboembolism, family history of thromboembolism, previous pulmonary embolism, family history of pulmonary embolism, Crohn's disease diagnosis, currently taking anti-hypertensive medication, previous orthopedic injury in the past 6-months, orthopedic surgery in the past 6-months, or a measured body mass index $>35 \mathrm{~kg} / \mathrm{m}^{2}$.

\section{Hypoxia}

Similar to previous work [4, 30, 31], hypoxia was induced using the Hypoxic Training System (Everest Summit II Hypoxic Generator, New York, USA). For the two experimental conditions, participants rested in a seated position with a mask affixed over their mouth and nose (for 30min). The hypoxic gas mixtures were generated through tubes that are connected to a breathing mask. The hypoxic condition involved breathing a gas mixture involving $\mathrm{FIO}_{2}$ of $12 \%$. 
Throughout this 30-min period, peripheral oxygen saturation $\left(\mathrm{SpO}_{2}\right)$ was measured via a pulse oximeter (Zacurate, $500 \mathrm{BL}$ ) placed on the right index finger. During the $30 \mathrm{~min}$ period, and every $5 \mathrm{~min}$, we also measured the participants' perceptual responses (for safety reasons) using the 2018 Lake Louise Scoring System (LLSS). This questionnaire evaluates four symptoms (headache, gastrointestinal symptoms, fatigue/weakness, and dizziness/lightheadedness). Four items were assessed for each of these categories, with response options of 0 (no symptoms) to 3 (severe symptoms). The hypoxia exposure ceased if the total LLSS score was 3 or higher. Notably, no participants had a LLSS score of 3 or higher.

\section{Memory function}

Identical to a previous experiment [4], on a desktop computer, participants completed a memory interference task using the $\mathrm{AB} / \mathrm{AC}$ paradigm. As noted below, two main memory outcomes were evaluated, including cued-recall and memory interference (proactive and retroactive). Participants were exposed to List 1 (AB-DE), which consists of eight word pairs (e.g., Beaver - Velvet) and then exposed to the Cued Recall List 1 where they recalled the missing word from the word pair (e.g., Beaver - __ ). Participants then completed a 20 second distractor task (i.e., mathematical problems) and then were exposed to List 2 (AC-FG), which consisted of eight-word pairs with some overlap of A words (e.g., Beaver - Parcel). After another 20 second distractor, participants were exposed to the Cued Recall of List 2 and attempted to recall the missing word from the word pair. Lastly, a final 20 second distractor was implemented, and participants then completed the Modified Modified Free Recall (MMFR) word list where they recalled a combined list of List 1 and List 2. The MMFR consists of all of the previously learned word pairs where some have only one missing word (DE, FG) and others have two missing word pair associations (AB, AC) (e.g., Circle Meadow, Beaver - Velvet and Parcel).

Using the participant's MMFR results, the number of correctly recalled words from the subset of words (AB, DE are within List 1 and AC, FG are within List 2) was calculated. Using the cued-recall results from List 1 and 2, proactive interference is measured by subtracting the percentage of correctly recalled FG pairs from the percentage of AC pairs (e.g., AC - FG) and retroactive interference is calculated by subtracting the percentage of correctly recalled $\mathrm{DE}$ pairs from the percentage of $\mathrm{AB}$ pairs (e.g., $\mathrm{AB}-\mathrm{DE}$ ). For both proactive and retroactive interference scores, lower values are indicative of an interference effect.

See Table 2 for an example of the memory interference assessment.

\section{Exercise protocol}

Participants exercised (moderate-intensity) for 20 -min on a treadmill at $50 \%$ of heart rate reserve (HRR). The HRR equation is:

$$
\mathrm{HRR}=\left[\left(\mathrm{HR}_{\max }-\mathrm{HR}_{\text {rest }}\right) * \% \text { intensity }\right]+\mathrm{HR}_{\text {rest }}
$$

To calculate $\mathrm{HR}_{\text {rest }}$, participants sat quietly for $3 \mathrm{~min}$ and $\mathrm{HR}$ was recorded from a Polar $\mathrm{HR}$ monitor at minute 3 . To estimate $\mathrm{HR}_{\max }$, we calculated the participants estimated $\mathrm{HR}_{\max }$ from 5 commonly used equations to estimate $\mathrm{HR}_{\max }$. We then took the average of these 5 estimates and used this average in the above $\mathrm{HRR}$ equation. The $5 \mathrm{HR}_{\max }$ equations that were used include:

Fox [32]: 220-age

Astrand [33]: $216.6-\left(0.84^{\star}\right.$ age $)$ 
Table 2. Memory interference protocol

\begin{tabular}{|c|c|c|c|c|}
\hline $\begin{array}{l}\text { Study Set } 1: \\
\text { AB, DE }\end{array}$ & $\begin{array}{l}\text { Cued Recall 1: } \\
\text { A_, D_- }\end{array}$ & $\begin{array}{l}\text { Study Set 2: } \\
\text { AC, FG }\end{array}$ & $\begin{array}{l}\text { Cued Recall 2: } \\
\text { A_, F__ }\end{array}$ & $\begin{array}{c}\text { MMFR: } A_{-}-D_{-} \\
\quad \mathrm{F}_{-}-\end{array}$ \\
\hline Baby Hunter & Spider & Forest City & Arrow & Baby \\
\hline Supper Sheriff & Forest & Arrow Theater & Forest & Cherry \\
\hline Shoe Movie & Baby & Baby Salad & Tiger & Arrow \\
\hline Spider Finger & Cherry & Tiger Hotel & Supper & Supper \\
\hline Monkey Garden & Monkey & Monkey Engine & Lady & Tiger \\
\hline Forest Battle & Supper & Lady Butter & Cannon & Shoe \\
\hline Cherry Money & Apple & $\begin{array}{l}\text { Cannon } \\
\text { Hammer }\end{array}$ & Baby & Monkey \\
\hline \multirow[t]{5}{*}{ Apple Grass } & Wedding & Supper Jacket & Monkey & Lady \\
\hline & & & & Spider \\
\hline & & & & Forest \\
\hline & & & & Apple \\
\hline & & & & Cannon \\
\hline
\end{tabular}

Tanaka et al. [34]: $208-\left(0.7^{\star}\right.$ age $)$

Gellish et al. [35]: $207-\left(0.7^{\star}\right.$ age $)$

Gulati et al. [36]: $206-\left(0.88^{\star}\right.$ age $)$

Throughout the treadmill exercise, HR was continuously monitored using a Polar HR monitor, and HRs will be recorded every $5 \mathrm{~min}$.

\section{Statistical analyses}

JASP (v. 0.10; Amsterdam, The Netherlands) statistical software was utilized to analyze the data. For the cued-recall results, a two-factor repeated measures ANOVA was employed, including a List factor (Recall 1 and Recall 2 of List 1 and List 2, respectively) and a condition factor (exercise then hypoxia and hypoxia alone). For the cued-recall memory results, main effects for list and condition, and list by condition interaction, were evaluated. For the memory interference results, a two-factor repeated measures ANOVA was employed, including factors for memory interference (proactive and retroactive) and condition (exercise then hypoxia and hypoxia alone). For the memory interference results, main effects for memory interference and condition, as well as condition by memory interference interaction, were evaluated. Two-factor (condition and time) repeated measures ANOVAs were also employed for the heart rate and peripheral oxygen saturation data. Statistical significance was set at an alpha of 0.05 . Eta squared $\left(\eta^{2}\right)$ was calculated as an effect size estimate.

\section{RESULTS}

Table 3 displays the characteristics of the sample. Participants, on average, were approximately 21 years and were predominately female (76.9\%), non-Hispanic white $(84.6 \%)$, and undergraduate $(88.5 \%)$. 
Table 3. Participant characteristics

\begin{tabular}{lcr}
\hline Variable & Point estimate & SD \\
\hline Age, mean years & 21.2 & 1.4 \\
\% Female & 76.9 & \\
Race-Ethnicity, \% & & \\
$\quad$ Non-Hispanic white & 84.6 & \\
Non-Hispanic black & 7.7 & \\
Other & 7.7 & 4.8 \\
Type of student & 88.5 & \\
Undergraduate & 11.5 & 100.2 \\
Graduate & 24.7 & \\
BMI, mean kg/m & \\
Mood & 2 & \\
MVPA, mean min/week & 133.9 & \\
\hline
\end{tabular}

Note. MVPA, moderate to vigorous physical activity (self-reported).

Fig. 1 displays the heart rates across the two experimental conditions. There was a statistically significant main effect for condition, $F(1,22)=168.51, P<0.001, \eta^{2}=0.18$, main effect for time, $F(6.82,150.02)=101.6, P<0.001, \eta^{2}=0.26$, and a statistically significant time by condition interaction, $F(8.25,181.67)=159.9, P<0.001, \eta^{2}=0.33$.

Fig. 2 displays the $\mathrm{SpO}_{2}$ across the two experimental conditions. There was a statistically significant main effect for time, $F(2.28,50.16)=94.82, P<0.001, \eta^{2}=0.62$, but no main effect for condition, $F(1,22)=0.17, P=0.68, \eta^{2}=0.00$, or time by condition interaction, $F(4.75$, 104.53) $=1.24, P=0.30, \eta^{2}=0.004$.

Table 4 displays the memory results across the two experimental conditions. For the cued-recall outcome, there was a statistically significant main effect for condition, $F(1,22)=$ 5.25, $P=0.03, \eta^{2}=0.04$, but no main effect for list, $F(1,22)=0.18, P=0.67, \eta^{2}=0.001$, or list by condition interaction, $F(1,22)=0.08, P=0.77, \eta^{2}=0.001$. The Exercise + Hypoxia condition

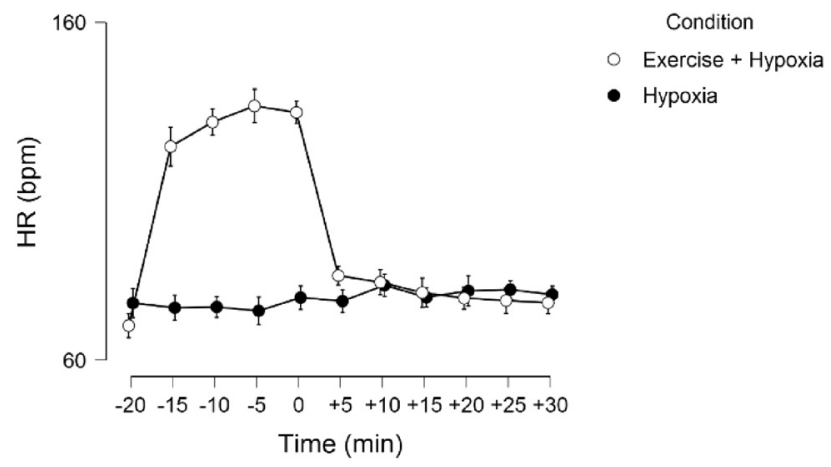

Fig. 1. Heart rate as a function of time and condition. Error bars represent $95 \%$ confidence intervals. Hypoxia exposure started at min 0 . In the Exercise + Hypoxia condition, exercise occurred from -20 to 0 


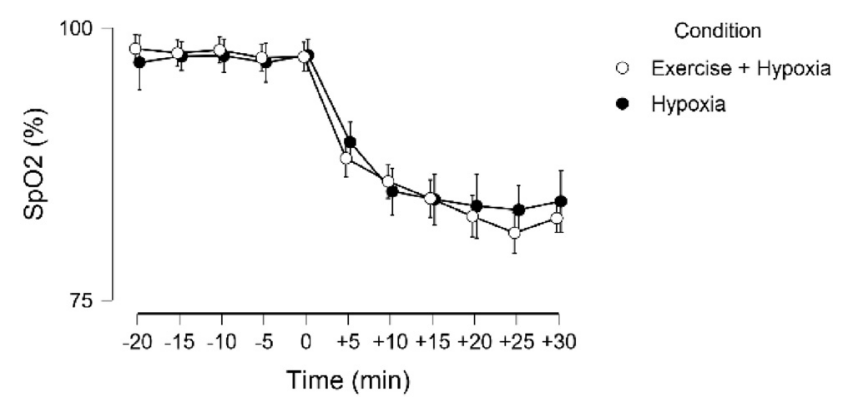

Fig. 2. Peripheral oxygen saturation as a function of time and condition. Error bars represent $95 \%$ confidence intervals. Hypoxia exposure started at min 0

Table 4. Memory results across the experimental conditions

\begin{tabular}{lcr}
\hline & Exercise & \\
Memory variable & Hypoxia & Hypoxia \\
Cued Recall 1, mean \# words & M (SD) & M (SD) \\
Cued Recall 2, mean \# words & $5.75(2.03)$ & $4.84(2.32)$ \\
PI, mean \% & $5.71(2.05)$ & $4.92(1.96)$ \\
RI, mean \% & $-10.87(24.80)$ & $-16.30(22.12)$ \\
\hline
\end{tabular}

Note. PI, proactive interference; RI, retroactive interference.

(cued-recall $1: \mathrm{M}=5.75, \mathrm{SD}=2.03$ and cued-recall $2: \mathrm{M}=5.71, \mathrm{SD}=2.05$ ) had greater cuedrecall memory than Hypoxia (cued-recall $1: \mathrm{M}=4.84, \mathrm{SD}=2.32$ and cued-recall $2: \mathrm{M}=4.92$, $\mathrm{SD}=1.96)$.

For the memory interference (proactive and retroactive) outcomes, there was a statistically significant main effect for memory interference, $F(1,22)=9.38, P=0.006, \eta^{2}=0.08$, but no main effect for condition, $F(1,22)=1.19, P=0.29, \eta^{2}=0.01$, or memory interference by condition interaction, $F(1,22)=0.04, P=0.84, \eta^{2}=0.001$. Retroactive interference scores (Exercise + Hypoxia condition: $\mathrm{M}=5.43, \mathrm{SD}=27.13$ and Hypoxia condition: $\mathrm{M}=-2.17$, $\mathrm{SD}=32.78$ ) were higher than proactive interference scores (Exercise + Hypoxia condition: $\mathrm{M}=-10.87, \mathrm{SD}=24.80$ and Hypoxia condition: $\mathrm{M}=-16.30, \mathrm{SD}=22.12$ ).

\section{DISCUSSION}

Although previous work has demonstrated that, independent of each other, acute exercise and acute hypoxia exposure may improve memory function and attenuate memory interference, no studies, to our knowledge, have evaluated whether combining an acute bout of moderate-intensity exercise with exposure to acute hypoxia may be more beneficial in improving memory function and reducing memory interference when compared to hypoxia alone. The present experiment demonstrates that when an acute bout of moderate-intensity exercise occurs prior to being exposed to acute hypoxia, cued-recall performance was enhanced when compared to 
hypoxia alone. Notably, we did not observe an additive effect of acute exercise and acute hypoxia on attenuating memory interference.

As stated, we anticipated that acute exercise combined with acute hypoxia would minimize a memory interference effect. This stemmed from our prior research demonstrating that both acute exercise and hypoxia alone can improve memory function and attenuate memory interference $[4,23,24]$. Our results partially align with our hypotheses, in that we observed an additive effect for cued-recall memory, but no effect on memory interference. The null memory interference effects may be explained by various factors. Perhaps exercise, simply, does not further enhance the effects of hypoxia on attenuating memory interference. Based on previous findings, it is possible that the exercise intensity implemented in the present experiment (i.e., moderate-intensity) was not sufficient to influence the effects of hypoxia on attenuating memory interference. Recent work provides evidence suggesting that when implemented prior to the memory task, high-intensity exercise may have favorable effects on episodic memory function [17]. Increased level of catecholamines, via high-intensity exercise, can enhance synaptic plasticity in the hippocampus [37]. Further, exercise-induced changes in norepinephrine can increase CREB transcription, and in turn, help maintain long-term potentiation [38]. Thus, given the fact that paired-associative learning is dependent on the hippocampus [39], higher exercise intensity may be better to attenuate memory interference. This is an area in need of future work. Another potential explanation for the null memory interference effect may be a result of the task used to evaluate memory interference. Although the $\mathrm{AB} / \mathrm{AC}$ memory interference paradigm is widely used to evaluate memory interference, interestingly, recent research has demonstrated that acute exercise can attenuate memory interference when using the RAVLT paradigm [24], an effect that was not replicated with the $\mathrm{AB} / \mathrm{AC}$ paradigm [40].

In addition to the potential intensity-specific effects of exercise, hypoxia-exercise temporality should carefully be considered in future work. Specifically, there may be a greater memory interference attenuation effect if an acute bout of exercise occurred during exposure to hypoxia. A recent narrative review demonstrated that acute exercise under brief hypoxia had a positive effect on cognitive function [41]. For example, when being exposed to a shorter duration of hypoxia, moderate-intensity exercise may increase cerebral blood flow and compensate for a decreased $\mathrm{SpO}_{2}$ [42]. Indeed, combined effects of acute exercise and hypoxia (moderate level) may activate synaptic plasticity via enhanced BDNF expression and its ensuing effects may facilitate cerebral neural activation, ultimately influencing cognitive improvement [31]. As such, it would be interesting to evaluate whether temporal modifications, such as acute exercise occurring during acute hypoxia, has an effect on attenuating memory interference.

A notable observation of our experiment was that acute exercise coupled with hypoxia exposure had a greater cued-recall memory performance than the hypoxia alone condition. We hypothesize that this effect is a result of the synergistic effects of acute exercise and acute hypoxia exposure on memory. As stated, both acute exercise and low dose hypoxia may upregulate neurophysiological markers (e.g., BDNF) thought to enhance hippocampal-dependent memory. As thoroughly discussed elsewhere [43], acute exercise is thought to elevate hippocampal BDNF levels via increased neural excitability in the hippocampus (from muscle spindle and vagus nerve stimulation) and inhibition of hippocampal histone deactylase (from production of D- $\beta$ hydroxybutyrate in the liver). Hypoxia has been shown to increase HIF-1alpha (hypoxia-inducible factor), which is known to act as a transcription factor to modulate the expression of BDNF $[44,45]$. 
In conclusion, previous work provides some suggestive evidence that acute hypoxia may help to reduce a memory interference effect [4]. Following this study, we are the first to evaluate the interactive effects of acute exercise and hypoxia on memory interference. The main findings from the present experiment are that acute exercise coupled with acute hypoxia may enhance cued-recall memory to a greater extent than hypoxia alone; however, no such effect was observed for memory interference. A limitation of this experiment is not including a normoxia condition. However, we intentionally chose not to include this condition as this has already been addressed in a previous experiment [4]. Notably, the specific research question of this experiment was whether acute exercise coupled with acute hypoxia would influence memory to a greater extent than hypoxia alone. Another limitation of this work includes the homogenous sample, which may reduce generalizability of our findings. Moreover, variation in physical activity (MVPA, $\mathrm{m}=133.9, \mathrm{SD}=100.2$ ) may influence aerobic capacity, potentially causing different exercise responses among participants. We are also limited by a lack of mechanistic measures (e.g., BDNF) and physiological responses (e.g., arterial oxygen saturation) to hypoxia exposure. Future work should also consider this paradigm while utilizing hypobaric conditions (vs. normobaric hypoxia model). Such suggestions will be important for subsequent work that aims to explore the interactive effects of engaging in acute exercise during exposure to hypoxic conditions on attenuating memory interference.

\section{ACKNOWLEDGMENTS}

We have no conflicts of interest. No funding was used to prepare this manuscript.

\section{REFERENCES}

1. Amann M, Kayser B. Nervous system function during exercise in hypoxia. High Alt Med Biol 2009; 10: 149-64.

2. Paola MD, Bozzali M, Fadda L, Musicco M, Sabatini U, Caltagirone C. Reduced oxygen due to high-altitude exposure relates to atrophy in motor-function brain areas. Eur J Neurol 2008; 15: 1050-7.

3. de Aquino Lemos V, Antunes HKM, dos Santos RVT, Lira FS, Tufik S, de Mello MT. High altitude exposure impairs sleep patterns, mood, and cognitive functions. Psychophysiology 2012; 49: 1298-306.

4. Loprinzi PD, Matalgah A, Crawford L, Yu JJ, Kong Z, Wang B, et al. Effects of acute normobaric hypoxia on memory interference. Brain Sci 2019; 9: 323.

5. McMorris T, Hale BJ, Barwood M, Costello J, Corbett J. Effect of acute hypoxia on cognition: a systematic review and meta-regression analysis. Neurosci Biobehav Rev 2017; 74: 225-32.

6. Dale EA, Ben Mabrouk F, Mitchell GS. Unexpected benefits of intermittent hypoxia: enhanced respiratory and nonrespiratory motor function. Physiology 2014; 29: 39-48.

7. Gozal D, Daniel JM, Dohanich GP. Behavioral and anatomical correlates of chronic episodic hypoxia during sleep in the rat. J Neurosci 2001; 21: 2442-50.

8. Lovett-Barr MR, Satriotomo I, Muir GD, Wilkerson JE, Hoffman MS, Vinit S, et al. Repetitive intermittent hypoxia induces respiratory and somatic motor recovery after chronic cervical spinal injury. J Neurosci 2012; 32: 3591-600.

9. Dale-Nagle EA, Hoffman MS, MacFarlane PM, Mitchell GS. Multiple pathways to long-lasting phrenic motor facilitation. Adv Exp Med Biol 2010; 669: 225-30. 
10. Lawley JS, Macdonald JH, Oliver SJ, Mullins PG. Unexpected reductions in regional cerebral perfusion during prolonged hypoxia. J Physiol 2017; 595: 935-47.

11. Bramham, CR, Messaoudi E. BDNF function in adult synaptic plasticity: the synaptic consolidation hypothesis. Prog Neurobiol 2005; 76: 99-125.

12. Cao L, Jiao X, Zuzga DS, Liu Y, Fong DM, Young D, et al. VEGF links hippocampal activity with neurogenesis, learning and memory. Nat Genet 2004; 36: 827-35.

13. Martens LK, Kirschner KM, Warnecke C, Scholz H. Hypoxia-inducible factor-1 (HIF-1) is a transcriptional activator of the TrkB neurotrophin receptor gene. J Biol Chem 2007; 282: 14379-88.

14. Bliss TV, Collingridge GL. A synaptic model of memory: long-term potentiation in the hippocampus. Nature 1993; 361: 31-9.

15. Yuan G, Nanduri J, Khan S, Semenza GL, Prabhakar NR. Induction of HIF-1 $\alpha$ expression by intermittent hypoxia: involvement of NADPH oxidase, $\mathrm{Ca}^{2+}$ signaling, prolyl hydroxylases, and mTOR. J Cell Physiol 2008; 217: 674-85.

16. Murray PS, Holmes PV. An overview of brain-derived neurotrophic factor and implications for excitotoxic vulnerability in the hippocampus. Int J Pept 2011; 2011: 654085.

17. Loprinzi PD. Intensity-specific effects of acute exercise on human memory function: considerations for the timing of exercise and the type of memory. Health Promot Perspect 2018; 8: 255.

18. Loprinzi PD, Edwards MK, Frith E. Potential avenues for exercise to activate episodic memory-related pathways: a narrative review. Eur J Neurosci 2017; 46: 2067-77.

19. Loprinzi PD. The effects of exercise on long-term potentiation: a candidate mechanism of the exercisememory relationship. OBM Neurobiol 2019; 3: 13.

20. Loprinzi PD, Ponce P, Frith E. Hypothesized mechanisms through which acute exercise influences episodic memory. Physiol Int 2018; 105: 285-97.

21. Frith E, Sng E, Loprinzi PD. Randomized controlled trial evaluating the temporal effects of high-intensity exercise on learning, short-term and long-term memory, and prospective memory. Eur J Neurosci 2017; 46: 2557-64.

22. Loprinzi PD, Blough J, Crawford L, Ryu S, Zou L, Li H. The temporal effects of acute exercise on episodic memory function: systematic review with meta-analysis. Brain Sci 2019; 9: 87.

23. Johnson L, Crawford L, Zou L, Loprinzi PD. Experimental effects of acute exercise in attenuating memory interference: considerations by biological sex. Medicina (Kaunas) 2019; 55: 331.

24. Loprinzi PD, Frith E, Crawford L. The effects of acute exercise on retroactive memory interference. Am J Health Promot 2020; 34: 25-31.

25. Crawford LK, Li H, Zou L, Wei GX, Loprinzi PD. Hypothesized mechanisms through which exercise may attenuate memory interference. Medicina (Kaunas) 2020; 56: 129.

26. Tsujii T, Komatsu K, Sakatani K. Acute effects of physical exercise on prefrontal cortex activity in older adults: a functional near-infrared spectroscopy study. Adv Exp Med Biol 2013; 765: 293-8.

27. Martínez MC, Villar ME, Ballarini F, Viola H. Retroactive interference of object-in-context long-term memory: role of dorsal hippocampus and medial prefrontal cortex. Hippocampus 2014; 24: 1482-92.

28. Guise KG, Shapiro ML. Medial prefrontal cortex reduces memory interference by modifying hippocampal encoding. Neuron 2017; 94: 183-92.

29. Koolschijn RS, Emir UE, Pantelides AC, Nili H, Behrens TE, Barron HC. The hippocampus and neocortical inhibitory engrams protect against memory interference. Neuron 2019; 101: 528-41.

30. Lei OK, Kong Z, Loprinzi PD, Shi Q, Sun S, Zou L, et al. Severe hypoxia does not offset the benefits of exercise on cognitive function in sedentary young women. Int J Environ Res Public Health 2019; 16: 1003. 
31. Sun S, Loprinzi PD, Guan H, Zou L, Kong Z, Hu Y, et al. The effects of high-intensity interval exercise and hypoxia on cognition in sedentary young adults. Medicina (Kaunas) 2019; 55: 43.

32. Fox 3rd SM. Physical activity and the prevention of coronary heart disease. Ann Clin Res 1971; 3: 404-32.

33. Astrand PO. Experimental studies of physical working capacity in relation to sex and age (dissertation). Copenhagen, Ejnar Munksgaard; 1952: 171 p.

34. Tanaka H, Monahan KD, Seals DR. Age-predicted maximal heart rate revisited. J Am Coll Cardiol 2001; 37: 153-6.

35. Gellish RL, Goslin BR, Olson RE, McDonald A, Russi GD, Moudgil VK. Longitudinal modeling of the relationship between age and maximal heart rate.Med Sci Sports Exerc 2007; 39: 822-9.

36. Gulati M, Shaw LJ, Thisted RA, Black HR, Bairey Merz CN, Arnsdorf MF. Heart rate response to exercise stress testing in asymptomatic women: the St. James women take heart project. Circulation 2010; 122: 130-7.

37. Gagnon SA, Wagner AD. Acute stress and episodic memory retrieval: neurobiological mechanisms and behavioral consequences. Ann N Y Acad Sci 2016; 1369: 55-75.

38. Roozendaal B, McGaugh JL. Memory modulation. Behav Neurosci 2011; 125: 797.

39. Yoon J, Seo Y, Kim J, Lee I. Hippocampus is required for paired associate memory with neither delay nor trial uniqueness. Learn Mem 2012; 19: 1-8.

40. Crawford L, Loprinzi PD. Effects of intensity-specific acute exercise on paired-associative memory and memory interference. Psych 2019; 1: 290-305.

41. Ando S, Komiyama T, Sudo M, Higaki Y, Ishida K, Costello JT, et al. The interactive effects of acute exercise and hypoxia on cognitive performance: a narrative review. Scand J Med Sci Sports 2020; 30: 384-98.

42. Curtelin D, Morales-Alamo D, Torres-Peralta R, Rasmussen P, Martin-Rincon M, Perez-Valera M, et al. Cerebral blood flow, frontal lobe oxygenation and intra-arterial blood pressure during sprint exercise in normoxia and severe acute hypoxia in humans. J Cereb Blood Flow Metab 2018; 38: 136-50.

43. Moore D, Loprinzi PD. Exercise influences episodic memory via changes in the hippocampal neurocircuitry by inducing long-term potentiation. Eur J Neurosci. https://doi.org/10.1111/ejn.14728, first published on March 31 (2020).

44. Helan M, Aravamudan B, Hartman WR, Thompson MA, Johnson BD, Pabelick CM, et al. BDNF secretion by human pulmonary artery endothelial cells in response to hypoxia. J Mol Cell Cardiol 2014; 68: 89-97.

45. Wiener CM, Booth G, Semenza GL. In vivo expression of mRNAs encoding hypoxia-inducible factor 1. Biochem Biophys Res Commun 1996; 225: 485-8. 